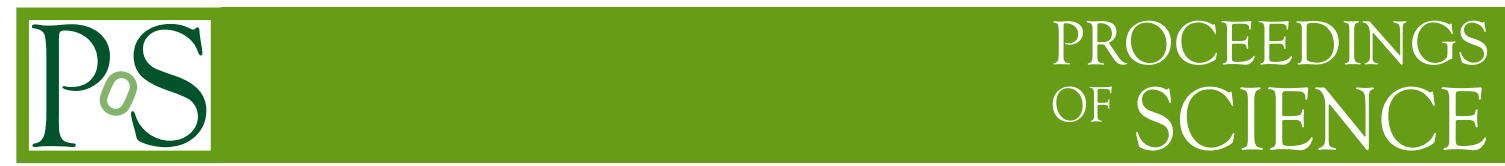

\title{
Nonlinear $k_{t}$ factorisation: recent progress and perspectives
}

\section{Wolfgang Schäfer*}

Institute of Nuclear Physics PAN, ul. Radzikowskiego 152, 31-342 Krakow, Poland

E-mail: wo.schaefer@fz-juelich.de

\begin{abstract}
Hard scattering in a strongly absorptive regime requires a novel nonlinear $k_{\perp}$-factorization. Here we discuss two recent developments: firstly the evaluation of radiative corrections to single particle spectra, and secondly an extension of the formalism to address topological cross sections and unitarity cutting rules.
\end{abstract}

Diffraction 06, International Workshop on Diffraction in High-Energy Physics September 5-10, 2006

Adamantas, Milos island, Greece

${ }^{*}$ Speaker. 


\section{Introduction}

Within perturbative QCD (pQCD) at high energies it is by now well understood that an adequate treatment should account explicitly for the transverse-momentum degrees of freedom of the participating partons. Specifically in the high-energy limit, this is accomplished by the socalled $k_{\perp}$-factorization which ultimately goes back to works on the Balitsky-Fadin-Kuraev-Lipatov (BFKL) equation for the small- $x$ evolution of the unintegrated glue ([1], for recent reviews on $k_{\perp}$ factorization and more references see [2,3]). An underlying assumption of the phenomenology based on (linear-) $k_{\perp}$-factorization is that it is sufficient to consider only single gluon (reggeon) exchange in the parton-target interaction. Certainly, this can only be warranted in a finite energy range, although perhaps covering most of the small- $x$ domain of $\gamma^{*} p$ collisions at HERA. Indeed, in a color-dipole picture [4], HERA data on diffractive deep-inelastic scattering imply, that for color dipoles $\sigma_{e l} / \sigma_{t o t} \ll 0.5$, that is unitarity constraints must be weak. Still, eventually the $s-$ channel partial waves of single-gluon exchange will overshoot unitarity bounds, and the inclusion of multiple gluon/reggeon exchanges associated with absorptive/unitarity corrections is mandatory. Although some semi-quantitative phenomenology exists (e.g.[5]), a systematic theory for proton targets is absent. Here heavy nuclei as targets offer a welcome testing ground for unitarity effects. Multiple gluon exchanges are enhanced by the large thickness of a heavy target nucleus, and one can concentrate on those unitarity corrections which grow with the size of the target $A$. From a different viewpoint [6], the opacity of heavy nuclei generates a new large scale $Q_{A}^{2} \propto T_{A}(\mathbf{b})$, the saturation scale, where $T_{A}(\mathbf{b})$ is the nuclear thickness (see e.g. [7] for a review and references). Especially in view of present and future programs on forward physics at RHIC and LHC, the fate of $k_{\perp}$ factorization in a regime of strong nuclear absorption is an important issue.

For illustration, consider the production of dijets in the virtual photon fragmentation region in $\gamma^{*}$-proton collisions. Here the dominant contribution comes from the $q \bar{q}$ final states and can be viewed as a hard photon-gluon fusion $\gamma^{*} g_{t} \rightarrow q \bar{q}$. While in the familiar collinear factorization the final state is a pair of back-to-back jets, in the lowest order of $k_{\perp}$-factorization the quark and antiquark jets are decorrelated in azimuth, and the distribution in the decorrelation momentum maps out the proton's unintegrated glue [8]:

$$
\frac{d \sigma\left(\gamma^{*} \rightarrow q \bar{q}\right)}{d z d^{2} \mathbf{K} d^{2} \mathbf{p}} \propto f(x, \mathbf{K})|\psi(z, \mathbf{p})-\psi(z, \mathbf{p}-\mathbf{K})|^{2}
$$

Here $\mathbf{p}$ is the transverse momentum of the quark-jet, $z$ is the fraction of the photon's lightcone momentum carried by the quark, and the transverse momentum $\mathbf{K}$ transferred by the gluon quantifies the azimuthal decorrelation of the $q \bar{q}$-system. Finally $\psi(z, \mathbf{p})$ is the momentum space lightconewavefunction (LCWF) for the transition $\gamma^{*} \rightarrow q \bar{q}$, and $f(x, \mathbf{K}) \propto \mathbf{K}^{-4} \partial G(x, \mathbf{K}) / \partial \log \left(\mathbf{K}^{2}\right)$ denotes the unintegrated gluon distribution of the proton target, which e.g. is directly proportional to the amplitude of hard diffractive dijet production [9]. When addressing the same observable in deep inelastic scattering (DIS) off nuclear targets [10], using a nuclear unintegrated glue that fulfills the same proportionality to hard diffractive amplitudes [11], we found that the linear $k_{\perp}-$ factorization is completely broken. The spectrum of dijets off nuclei is an involved nonlinear functional with little resemblance ${ }^{1}$ of the free-nucleon result eq. (1.1). It incorporates distinct

\footnotetext{
${ }^{1}$ See eq.(4.1) below.
} 
physical effects, such as the coherent nuclear distortion of the $q \bar{q} \mathrm{LC}-\mathrm{WF}$ as well as incoherent transverse-momentum broadening through intranuclear multiple scattering, which arise from the same unitarity-driven dynamics. We dubbed the emerging momentum-space formalism Nonlinear $k_{\perp}$ Factorization, and subsequently developed the description of other relevant hard subprocesses, such as $\pi \rightarrow q \bar{q}, q \rightarrow q g, g \rightarrow g g, g \rightarrow q \bar{q}[12,13,14]$. In this contribution we will address the most recent developments: first, the evaluation of virtual radiative corrections to single-jet observables, and the small- $x$ evolution of the nuclear unintegrated glue from the $s$-channel unitarity relations [15], and second an extension to more refined observables, such as topological cross sections and the related unitarity rules [16] of Abramovsky-Gribov-Kancheli (AGK [17]) type. This will include prolegomena to a Reggeon Field Theory (RFT) interpretation of the nonlinear $k_{\perp}$-factorization results. The breaking of linear $k_{\perp}$-factorization has also been reported in other approaches, we mention [18].

\section{Nuclear unintegrated glue $[10,11]$, and its evolution [15]}

Swiftly moving partons propagate along fixed impact parameter trajectories, and their impact parameter is conserved in the interaction with the target $A$. Consider a parton $a$ in an arbitrary color representation, and let $\mathrm{S}_{a}\left(\mathbf{b}_{a}\right)$ be the $S$-matrix of the (bare) parton-target interaction in the target rest frame at an impact parameter $\mathbf{b}_{a}$. Then, the $S$-matrix for the $a \bar{a}$ color-dipole of size $\mathbf{r}=\mathbf{b}_{a}-\mathbf{b}-a$ scattering at $\mathbf{b}$

$$
\mathrm{S}_{a-}(\mathbf{b}, \mathbf{r})=\frac{\left\langle A\left|\operatorname{Tr}\left[\mathrm{S}_{a}\left(\mathbf{b}_{a}\right) \mathbf{S}_{a}^{\dagger}\left(\mathbf{b}_{a}-\mathbf{r}\right)\right]\right| A\right\rangle}{\langle A|\operatorname{Tr} I| A\rangle}
$$

can be evaluated by standard Glauber-Gribov $[19,20]$ techniques at a boundary value $x_{A}=\left(2 R_{A} m_{N}\right)^{-1}$ $\left(R_{A}\right.$ is the nuclear radius, $m_{N}$ the nucleon mass) as $\mathrm{S}_{a^{-}}(\mathbf{b}, \mathbf{r})=\exp \left[-\frac{1}{2} \sigma_{a}-\left(x_{A}, \mathbf{r}\right) T_{A}(\mathbf{b})\right]$, and serves to define the nuclear unintegrated gluon distribution $\phi\left(\mathbf{b}, x_{A}, \mathbf{p}\right)$

$$
\Phi\left(\mathbf{b}, x_{A}, \mathbf{p}\right)=\int \frac{d^{2} \mathbf{r}}{(2 \pi)^{2}} \mathbf{S}_{a}-(\mathbf{b}, \mathbf{r}) \exp [-i \mathbf{p r}]=\exp \left[-v_{A}(\mathbf{b})\right] \boldsymbol{\delta}^{(2)}(\mathbf{p})+\phi\left(\mathbf{b}, x_{A}, \mathbf{p}\right) .
$$

Using its Glauber-Gribov relation to the free-nucleon color-dipole cross section $\sigma\left(x_{A}, \mathbf{r}\right)_{a^{-} a}$ one derives a convenient representation of the coherent nuclear glue $\phi\left(\mathbf{b}, x_{A}, \mathbf{p}\right)$ in terms of multiple convolutions $f^{(j)}(\mathbf{p})=\left(f \otimes f^{(j-1)}\right)(\mathbf{p})$ of the free-nucleon unintegrated glue $f(\mathbf{p}): \phi\left(\mathbf{b}, x_{A}, \mathbf{p}\right)=$ $\sum w_{j}\left(v_{A}(\mathbf{b})\right) f^{(j)}(\mathbf{p})$. Here the Poisson weights ${ }^{2} w_{j}=\exp \left[-v_{A}(\mathbf{b})\right] v_{A}^{j}(\mathbf{b}) / j$ ! represent the probability to find $j$ overlapping nucleons at impact parameter $\mathbf{b}$. The salient features of $\phi\left(\mathbf{b}, x_{A}, \mathbf{p}\right)$ can be briefly summarized: first, for realistic free-nucleon input and heavy nuclei there emerges a saturation scale $Q_{A}^{2}\left(x_{A}\right) \sim 0.8 \div 1 \mathrm{GeV}^{2}$, second, at large $\mathbf{p}^{2}$ we have a Cronin-type antishadowing enhancement that is calculable parameter-free, third it furnishes a linear $k_{\perp}$-factorization of inclusive DIS, forward single-jets in DIS and diffractive dijet amplitudes. To study evolution to small $x \ll x_{A}$, one has to follow the strategy of [21], and integrate out the effect of an additional s-channel gluon, schematically:

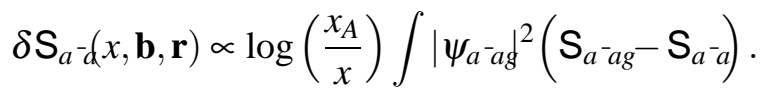

\footnotetext{
${ }^{2}$ The nuclear opacity $v_{A}(\mathbf{b})=\frac{1}{2} \sigma_{0}\left(x_{A}\right) T_{A}(\mathbf{b})$ involves the dipole cross section for large color dipoles $\sigma_{0}\left(x_{A}\right)$ and the nuclear thickness function $T_{A}(\mathbf{b})$.
} 


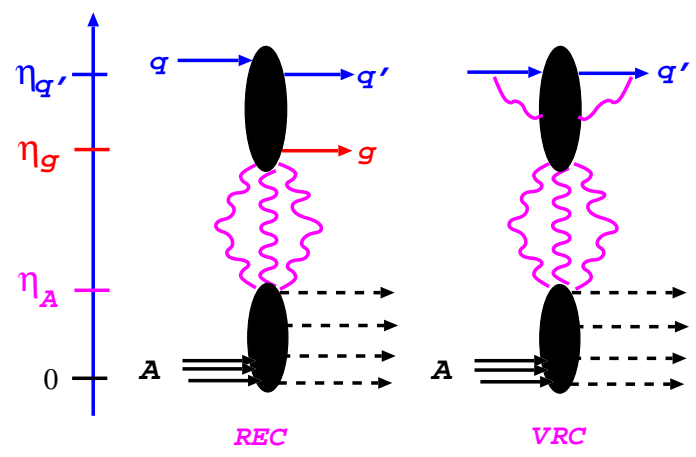

Figure 1: The real emission (REC) and virtual radiation (VRC) contributions to the spectrum of leading quark jets in inclusive production off nuclei. The (pseudo)rapidity of radiation correction gluons runs between the quark jet rapidity and the nuclear boundary condition rapidity $\eta_{A}=\log \left(1 / x_{A}\right)$, where $x_{A}$ is defined in the text.

This produces the first step of the small- $x$ evolution, a closed form of which does not exist, and is equivalent to the first step of the Balitsky-Kovchegov (BK) equation [22]. Using the relation (2.2) one may translate (2.4) to momentum space. Concentrating on color-triplet partons (quarks), one would obtain [15]

$$
\delta \phi(\mathbf{b}, x, \mathbf{p})=\log \left(\frac{x_{A}}{x}\right)\left[\left(\mathscr{K}_{B F K L} \otimes \phi\right)\left(\mathbf{b}, x_{A}, \mathbf{p}\right)+\mathscr{Q}[\phi]\left(\mathbf{b}, x_{A}, \mathbf{p}\right)\right] .
$$

Here we decomposed the nuclear evolution into the linear, BFKL-evolution piece, $\left(\mathscr{K}_{B F K L} \otimes\right.$ $\phi)\left(\mathbf{b}, x_{A}, \mathbf{p}\right)=C_{A} \alpha_{S}\left(2 \pi^{2}\right)^{-2} \int d^{2} \mathbf{q}\left[2 K(\mathbf{p}, \mathbf{p}-\mathbf{q}) \phi\left(\mathbf{b}, x_{A}, \mathbf{q}\right)-\phi\left(\mathbf{b}, x_{A}, \mathbf{p}\right) K(\mathbf{q}, \mathbf{q}-\mathbf{p})\right]$, and the genuinely nonlinear component represented by the quadratic functional ${ }^{3}$

$$
\begin{aligned}
\mathscr{Q}[\phi]\left(\mathbf{b}, x_{A}, \mathbf{p}\right) & =\int d^{2} \mathbf{q}_{1} d^{2} \mathbf{q}_{2} \phi\left(\mathbf{b}, x_{A}, \mathbf{q}_{1}\right)\left\{\left[K\left(\mathbf{p}+\mathbf{q}_{1}, \mathbf{p}+\mathbf{q}_{2}\right)-K\left(\mathbf{p}, \mathbf{p}+\mathbf{q}_{1}\right)\right.\right. \\
& \left.\left.-K\left(\mathbf{p}, \mathbf{p}+\mathbf{q}_{2}\right)\right] \phi\left(\mathbf{b}, x_{A}, \mathbf{q}_{2}\right)-\phi\left(\mathbf{b}, x_{A}, \mathbf{p}\right)\left[K\left(\mathbf{q}_{1}, \mathbf{q}_{1}+\mathbf{q}_{2}+\mathbf{p}\right)-K\left(\mathbf{q}_{1}, \mathbf{q}_{1}+\mathbf{p}\right)\right]\right\} .
\end{aligned}
$$

The latter generates a higher-twist contribution to the large-p tail of the nuclear collective glue, but it does not exhaust the nuclear higher twists, recall that the boundary condition itself contains substantial - but anti-shadowing - higher twist contributions.

\section{Forward jets from the breakup of valence quarks [15]}

Consider now the production of jets/high $p_{\perp}$ particles at forward rapidities, i.e. in the beam fragmentation region in a high energy proton-nucleus collision. The dominant beam partons are evidently valence quarks. The density of valence quarks is a steep function for $x \rightarrow 1$, and the production of high $p_{\perp}$ forward partons must be highly sensitive to their energy loss. Here we discuss the perturbative, radiative energy loss that originates from the transition $q \rightarrow q g$. The dynamics of partons with rapidities $\eta>\eta_{A}=\log \left(1 / x_{A}\right)$, proceeds coherently over the whole nucleus (for some notation see Fig. 1). Notice that in addition to the coherent excitation $q \rightarrow q g$, in the $k_{\perp}$

\footnotetext{
${ }^{3}$ here, for perturbative large $\mathbf{p}_{i}, K\left(\mathbf{p}_{1}, \mathbf{p}_{2}\right)=\left(\mathbf{p}_{1}-\mathbf{p}_{2}\right)^{2} / \mathbf{p}_{1}^{2} \mathbf{p}_{2}^{2}$. For a regularized form valid also at small $\mathbf{p}_{i}$, see [15].
} 
factorization one must also add the quasielastic scattering of the beam quark, where its transverse momentum is balanced by (mini-) jets with rapidities $\eta<\eta_{A}$. To quantify the $p_{\perp}$-dependent radiative energy loss, one must equally evaluate the radiative correction to the quasielastic scattering, i.e. the virtual corrections in terms of fast $\eta>\eta_{A}$ parton degrees of freedom depicted as VRC in Fig. 1. The calculation of radiative corrections, real as well as virtual is most expediently performed in impact parameter space. To this end, one expands the LCWF of the beam quark into its bare quark and quark-gluon components. By the conservation of impact parameters, the $S$-matrix acts on the physical quark state in a simple form:

$$
S\left|q_{p h y s}(\mathbf{b})\right\rangle=\left[S_{q}(\mathbf{b})+\delta S_{q}(\mathbf{b})\right]\left|q_{p h y s}(\mathbf{b})\right\rangle+\left[S_{q}\left(\mathbf{b}_{q}^{\prime}\right) S_{g}\left(\mathbf{b}_{g}\right)-S_{q}(\mathbf{b})\right] \psi_{q g}\left(z_{b}, \mathbf{b}_{q}^{\prime}-\mathbf{b}_{g}\right)\left|q\left(\mathbf{b}_{q}^{\prime}\right) g\left(\mathbf{b}_{g}\right)\right\rangle .
$$

Evaluation of two-parton and single-parton spectra from the excitation operator $S_{e x}=S_{q}\left(\mathbf{b}_{q}^{\prime}\right) S_{g}\left(\mathbf{b}_{g}\right)$ $S_{q}(\mathbf{b})$ is discussed in detail in $[13,14]$. The important point is that $S_{e x}$ determines through the unitarity relation also the radiative correction $\delta S_{q}(\mathbf{b})$ to the quasielastic scattering of the quark, which reads:

$$
\delta S_{q}(\mathbf{b})=\int \mathscr{D}\left\{\mathbf{b}_{g}, z_{g}\right\}\left|\psi_{q g}\right|^{2}\left[S_{q}\left(\mathbf{b}_{q}^{\prime}\right) S_{g}\left(\mathbf{b}_{g}\right)-S_{q}(\mathbf{b})\right] .
$$

It is important to stress, that the LCWF/color-dipole S-matrix approach is fully general in that it applies equally to the single-gluon exchange encountered on the free nucleon as well as to the multigluon exchanges relevant to strongly absorbing nuclei. Also notice, that we do not require the multi-Regge type kinematics of $\eta_{g} \ll \eta_{q}$, but allow for an arbitrary rapidity spacing of scattered quark and the radiative gluon as is appropriate for the evaluation of the radiative energy loss. We skip the detailed form of the single-parton spectra, as well as the case of incident color-octet partons, and close this section with a remark on the small- $x$ evolution properties of the singlequark spectra. At the boundary value $x_{A}$ one can easily check that the latter is directly proportional to the target unintegrated glue, $d \sigma_{Q e l}\left(q \rightarrow q, x_{A}\right) / d^{2} \mathbf{b} d^{2} \mathbf{p}=\phi\left(\mathbf{b}, x_{A}, \mathbf{p}\right)$. The intriguing result is now that this relation holds, to the leading $-\log (1 / x)(\operatorname{LL}(1 / x))$ also after the radiative corrections, that is

$$
\frac{d \sigma_{Q e l}(q \rightarrow q, x)}{d^{2} \mathbf{b} d^{2} \mathbf{p}}=\phi\left(\mathbf{b}, x_{A}, \mathbf{p}\right)+\delta \phi(\mathbf{b}, x, \mathbf{p}),
$$

with the same $\delta \phi(\mathbf{b}, x, \mathbf{p})$ of eq.(2.4) that defines the first $\operatorname{LL}(1 / x)$ iteration of the nonlinear small- $x$ evolution of the nuclear unintegrated glue. We recall also the earlier result [13], that the spectrum of midrapidity gluons is linearly factorizable in terms of the nuclear unintegrated glue. All of this suggests a deeper role of the coherent nuclear glue in terms of a to be developed RFT description. Some of these aspects we shall address in the following.

\section{Topological cross sections and unitarity cutting rules [16]}

In linear $k_{\perp}$ factorization the partonic transitions $a \rightarrow b c$ of interest proceed via single gluon exchange and leave the recoiling target nucleon debris in a color octect state. There is an obvious connection between the unintegrated gluon distribution and a cut (BFKL-) Pomeron. For example, the quasi-elastic scattering of parton $a$ with a color excited nucleon in the final state is $d \sigma_{Q e l} / d^{2} \mathbf{p}=C_{a} / 2 C_{F} f(x, \mathbf{p})$, where $C_{a}, C_{F}$ are color Casimirs and the nuclear unintegrated glue $f$ represents the coupling of the color-octet $t$-channel exchange to the nucleon. The situation is more 
involved for the heavy nucleus target. On the beam side, during its coherent interaction with the nucleus the $b c$ parton system will evolve over all possible color multiplets in the relevant product representation space. The at first sight formidable problem in color algebra can be solved [14] using a technique originally due to Zakharov, where by concentrating on the $b c \bar{b}^{\prime} \bar{c}^{\prime}$-density matrix one deals with a four-parton system in an overall color-singlet state. The intranuclear evolution problem is then solved by a color-coupled channel four-body $S$-matrix $S(\mathbf{C})=\exp \left[-\frac{1}{2} \Sigma^{(4)}(\mathbf{C}) T_{A}\right]$ (here $\mathbf{C}$ collectively denotes the relevant impact parameters). The building block is the color-dipole cross section operator $\Sigma^{(4)}(\mathbf{C})$ for the $b c \bar{b}^{\prime} \bar{c}^{\prime}$ system for the free-nucleon target. It is a matrix in the space of possible color-singlet states $|R \bar{R}\rangle=\left|(b c)_{R} \otimes\left(\bar{b}^{\prime} \bar{c}^{\prime}\right)_{\bar{R}}\right\rangle$. The overall-singlet property of the $b c \bar{b}^{\prime} \bar{c}^{\prime}$-system implies that its matrix elements are infrared safe combinations of the free-nucleon color dipole cross section. It is important to distinguish the following aspects of color channel coupling: a) transitions of the $b c$-system between two multiplets, $R_{i} \rightarrow R_{j}$ of different dimensionality. While these transitions are in general suppressed in the number of colors $N_{c}$, a large number of final states in higher multiplets can overcome this suppression. The $t$-channel gluons that induce these transitions must be hard to resolve the color structure. Correspondingly, such color excitations can be treated perturbatively in an expansion, that is simultaneously a large $-N_{c} /$ hard scattering expansion. b) transitions of the $b c$-systems that are rotations within the same color multiplet $R_{i} \rightarrow R_{i}$. Notice that these can involve singlet, as well as octet exchange with a scattering center in the target. Such contributions need to be summed to all orders and place no restriction on the hardness of the t-channel gluons.

When viewed from the target side, after the scattering the nucleus will be left in a state with multiple color excited nucleons ${ }^{4}$. The higher the number of color excited nucleons, the higher will also be the multiplicity of particles produced in the target hemisphere. This clearly implies nonperturbative mechanisms for energy loss of the $b c$ system. In addition, this picture offers a perfect definition of cut Pomerons and connection with the RFT language: each and every color excited nucleon must be associated with a unitarity cut of a Pomeron exchanged between beam and target. From a technical point of view, the counting of color-octet nucleons is possible after a decomposition of the dipole cross section-operator $\Sigma^{(4)}(\mathbf{C})=\Sigma_{e l}^{(4)}(\mathbf{C})+\Sigma_{e x}^{(4)}(\mathbf{C})$ into an 'elastic' piece $\Sigma_{e l}^{(4)}$ that involves two-gluon color singlet exchange with the nucleon, and an excitation piece $\Sigma_{e x}^{(4)}$ that involves color-octet exchange with a nucleon ${ }^{5}$. We come to the implications on the unitarity cut interpretation of the nuclear unintegrated glue. Firstly, one would easily identify the cross section for $j$-fold quasielastic scattering of a parton $a$ as $d \sigma_{Q e l}^{(j)} / d^{2} \mathbf{p}=C_{a} / 2 C_{F} f^{(j)}(\mathbf{p})$. The familiar expansion of the nuclear unintegrated glue over multiple convolutions now takes a new interpretation as an expansion over unitarity cuts corresponding to final states with $j$ color excited nucleons: $\phi(\mathbf{b}, x, \mathbf{p})=\sum w_{j}\left(v_{A}(\mathbf{b})\right) d \sigma_{Q e l}^{(j)} / \sigma_{Q e l} d^{2} \mathbf{p}$. In the alternating sign expansion $\exp \left[-v_{A}(\mathbf{b})\right]=$ $\sum(-1)^{k} / k !\left[T_{A} / 2\right]^{k}\left[\int d^{2} \mathbf{p} f(\mathbf{p})\right]^{k}$ of the exponential entering $w_{j}$, the $k$-th order term corresponds precisely to an absorptive correction from $k$ uncut Pomeron exchanges. This suggests an interpretation of $\phi(\mathbf{b}, x, \mathbf{p})$ as a coupling of the absorbed gluon-Reggeon to the nucleus. Out of the many possible applications we give a brief description on the RFT-cut interpretation of forward-dijets in inelastic

\footnotetext{
${ }^{4}$ Note a certain similarity to the concept of wounded nucleons [23].

${ }^{5}$ Such decomposition is clearly infrared sensitive, but so is also the transition from cut Pomerons to fi nal state particle multiplicities.
} 
DIS - the counterpart of the free nucleon result eq.(1.1). We obtain the nonlinear $k_{\perp}$-factorization for topological cross sections of DIS followed by color excitation of $v$ nucleons ( $v$ cut pomerons) 6 :

$$
\begin{aligned}
& \frac{d \sigma_{v}\left(\gamma^{*} A \rightarrow\{q \bar{q}\}_{8} X\right)}{d^{2} \mathbf{b} d z d^{2} \mathbf{p} d^{2} \mathbf{K}}=\frac{T_{A}(\mathbf{b})}{(2 \pi)^{2}} \int_{0}^{1} d \beta \int d^{2} \kappa_{1} d^{2} \kappa_{2} d^{2} \kappa \delta\left(\mathbf{K}-\kappa_{1}-\kappa_{2}-\kappa\right) \\
\times & \frac{d \sigma_{Q e l}(\kappa)}{d^{2} \kappa}\left|\psi\left(\beta ; z, \mathbf{p}-\kappa_{1}\right)-\psi\left(\beta ; z, \mathbf{p}-\kappa_{1}-\kappa\right)\right|^{2} \\
\times & \sum_{j, k=0} \delta(v-1-j-k) w_{j}\left((1-\beta) v_{A}(\mathbf{b})\right) w_{k}\left((1-\beta) v_{A}(\mathbf{b})\right) \frac{d \sigma_{Q e l}^{(k)}\left(\kappa_{1}\right)}{\sigma_{Q e l} d^{2} \kappa_{1}} \cdot \frac{d \sigma_{Q e l}^{(j)}\left(\kappa_{2}\right)}{\sigma_{Q e l} d^{2} \kappa_{2}} .
\end{aligned}
$$

The color channel coupling effects manifest themselves in the presence of two different types of cut Pomerons, here they show up as $d \sigma_{Q e l}(\kappa) / d^{2} \kappa \propto f(\kappa)$, which derives from the singlet-tooctet transition of the $q \bar{q}$-pair, whereas $d \sigma_{Q e l}^{(k)}\left(\kappa_{1}\right) / \sigma_{Q e l} d^{2} \kappa_{1} \cdot d \sigma_{Q e l}^{(j)}\left(\kappa_{2}\right) / \sigma_{Q e l} d^{2} \kappa_{2}$ derive from color rotations of the $q \bar{q}$-pair in the color-octet state; quark and antiquark rescatter independently and contribute $j$, respectively $k$ color excited nucleons to the final state. The RFT structure is further explained in Fig. 2. When going over to topological cross sections for single-jet production, say, the single quark spectrum, interactions of the spectator anti-quark do not cancel but leave a Cheshire Cat Grin (CCG): the final state will contain color excited nucleons that derive from interactions of the antiquark. Amazingly, a novel multiplicity resummation, removes the CCG. The contribution from $k$ cut Pomerons can be isolated from

$$
\frac{d \sigma^{(k)}\left(\gamma^{*} A \rightarrow q X\right)}{d^{2} \mathbf{b} d z d^{2} \mathbf{p}}=\sum_{v>k} \frac{d \sigma_{v}\left(\gamma^{*} A \rightarrow q X\right)}{d^{2} \mathbf{b} d z d^{2} \mathbf{p}},
$$

which roughly corresponds to a re-summation over backward multiplicities $n_{B}>k\left\langle n_{B}\right\rangle_{p p}$.

As a consequence of two types of cut Pomerons, common doctrine from hadronic Glaubertype models [24] is entirely inapplicable in the pQCD realm. For example an expansion of the inelastic Glauber profile function for color dipoles

$$
\Gamma^{(i n e l)}=1-\exp \left[-\sigma(x, \mathbf{r}) T_{A}(\mathbf{b})\right]=\exp \left[-\sigma(x, \mathbf{r}) T_{A}(\mathbf{b})\right] \sum \frac{1}{v !}\left[\sigma(x, \mathbf{r}) T_{A}(\mathbf{b})\right]^{v}
$$

simply has no sensible unitarity interpretation at all. In particular it does not allow one to extract topological cross sections from the inclusive inelastic profile function. Additionally, relations on the double Pomeron contributions that are in widespread use as a unitarisation snake-oil, such as the cancellation of discontinuities

$$
\Delta_{2} \Gamma^{(i n)}(\mathbf{I I} / \mathbf{I P})+2 \Delta_{2} \Gamma^{(i n)}\left(\mathbf{I} / \mathbf{I} / \mathbf{H}^{\prime}\right)=0
$$

and the "AGK-ratios"

$$
\Delta_{2} \Gamma_{D}(\mathbf{I P I P}): \Delta_{2} \Gamma_{1}^{i n}(\mathbf{I H} / \mathbf{P P}): \Delta \Gamma_{2}^{i n}(\mathbf{I} / \mathbf{I P} /)=1:-4: 2,
$$

\footnotetext{
${ }^{6}$ Here $\beta$ is a dimensionless longitudinal depth of the nucleus, which arises because of the noncommutativity of $\Sigma_{e l}^{(4)}$ and $\Sigma_{e x}^{(4)}$, and $\psi(\beta, \ldots)$ denotes a LCWF coherently distorted over a slice of length $\beta$.
} 


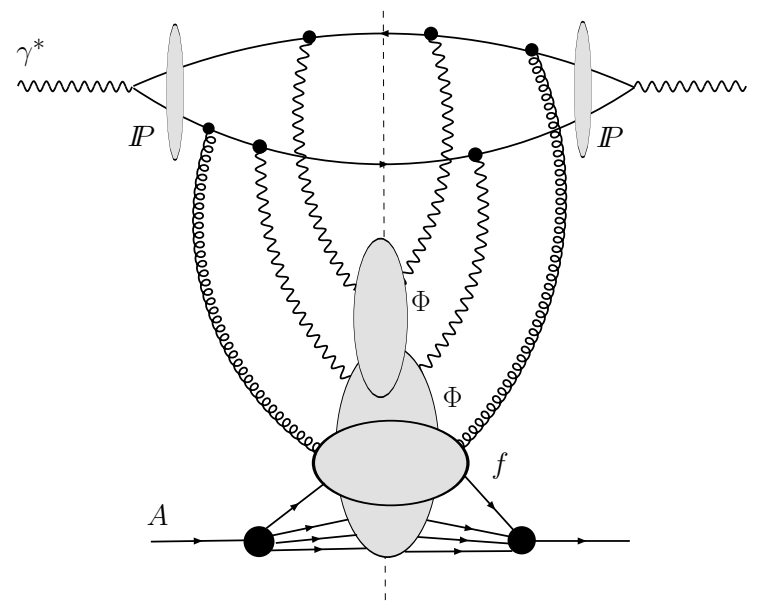

Figure 2: One of four diagrams representing the RFT unitarity cut for inelastic DIS in terms of two types of cut Pomerons. The curly lines represent gluons from the cut Pomeron II/ associated with the singlet to octet transition of the $q^{-} q$ pair, and couples to the target through the free-nucleon glue $f$. Zigzag-lines are associated with two cut nuclear Pomerons II/ ${ }_{r, A}$ associated with color rotations in the $q^{-} q$-octet channel. Their coupling through the target is through the nuclear unintegrated glue $\Phi$. When expanded out they give rise to the factors $\propto d \sigma_{Q e l}^{(k)}\left(\kappa_{1}\right) \cdot d \sigma_{Q e l}^{(j)}\left(\kappa_{2}\right)$ in eq. (4.1). Also indicated are the coherent distortions of the $\gamma^{*} \rightarrow q^{-} q$ LCWF through uncut Pomerons. Notice the cylinder/Mandelstam structure of the cut. The coupling of multiple Pomerons is local in rapidity and involves no branching tree of triple Pomeron interactions/fan diagrams.

take no account of the intricacies of color channel coupling (two types of cut Pomerons) and the subtleties of spectator interactions. See [16] for the -infrared sensitive- relations that replace them. Gribov's unitarity relation $\Delta_{2} \Gamma_{\text {tot }}(\mathbf{I P I P})=-\Delta_{2} \Gamma_{\text {Diffractive }}(\mathbf{I P I P})$ between the shadowing correction to the total cross section and the diffractive profile function is model independent, and remains of course valid.

\section{Summary}

Hard scattering in a regime of strong opacity requires a novel, nonlinear $k_{\perp}$-factorization. Here we would not reiterate our points which we made clear enough in the main text, rather we end with an outlook: there remains a long shopping list of phenomenological applications connected to the production of forward high $p_{\perp}$-particles/jets, such as: nonperturbative and perturbative quenching, its centrality dependence, forward-backward correlations between hard production in the beamand multiplicities in the backward hemispheres, azimuthal decorrelations, etc, and we look forward to practical applications. On the theoretical side a study of multipomeron couplings and the RFT-interpretation of the small- $x$ evolution of the coherent nuclear glue are to be ordered for Diffraction2008.

It is a pleasure to thank the organisers, especially Igor Ivanov and Alessandro Papa, for their efforts, and for invitation to this exciting workshop. 


\section{References}

[1] E. A. Kuraev, L. N. Lipatov and V. S. Fadin, The Pomeranchuk Singularity In Nonabelian Gauge Theories, Sov. Phys. JETP 44, 443 (1976) [Zh. Eksp. Teor. Fiz. 71, 840 (1976)]; Multi - Reggeon Processes In The Yang-Mills Theory, Sov. Phys. JETP 45, 199 (1977) [Zh. Eksp. Teor. Fiz. 72, 377 (1977)]; I. I. Balitsky and L. N. Lipatov, The Pomeranchuk Singularity In Quantum Chromodynamics, Sov. J. Nucl. Phys. 28, 822 (1978) [Yad. Fiz. 28, 1597 (1978)].

[2] B. Andersson et al. [Small x Collaboration], Small x phenomenology: Summary and status, Eur. Phys. J. C 25, 77 (2002) [arXiv:hep-ph/0204115]; J. R. Andersen et al. [Small x Collaboration], Small $x$ phenomenology: Summary and status 2002, Eur. Phys. J. C 35, 67 (2004) [arXiv:hep-ph/0312333]; J. R. Andersen et al. [Small x Collaboration], Small x phenomenology: Summary of the 3rd Lund small $x$ workshop in 2004, arXiv:hep-ph/0604189.

[3] A. Szczurek, From unintegrated gluon distributions to particle production in nucleon nucleon collisions at RHIC energies, Acta Phys. Polon. B 34, 3191 (2003) [arXiv:hep-ph/0304129]. J. Collins and H. Jung, Need for fully unintegrated parton densities, arXiv:hep-ph/0508280.

[4] N. N. Nikolaev and B. G. Zakharov, Colour Transparency And Scaling Properties Of Nuclear Shadowing In Deep Inelastic Scattering, Z. Phys. C 49, 607 (1991); N. Nikolaev and B. G. Zakharov, Pomeron structure function and diffraction dissociation of virtual photons in perturbative QCD, Z. Phys. C 53, 331 (1992).

[5] V. Barone, M. Genovese, N. N. Nikolaev, E. Predazzi and B. G. Zakharov, Unitarization of structure functions at large 1/x, Phys. Lett. B 326, 161 (1994) [arXiv:hep-ph/9307248].

[6] A. H. Mueller, Parton saturation at small $x$ and in large nuclei, Nucl. Phys. B 558 (1999) 285 [arXiv:hep-ph/9904404].

[7] J. Jalilian-Marian and Y. V. Kovchegov, Saturation physics and deuteron gold collisions at RHIC, Prog. Part. Nucl. Phys. 56, 104 (2006) [arXiv:hep-ph/0505052]; R. Venugopalan, The color glass condensate: An overview, Eur. Phys. J. C 43, 337 (2005) [arXiv:hep-ph/0502190].

[8] A. Szczurek, N. N. Nikolaev, W. Schäfer and J. Speth, Mapping the proton unintegrated gluon distribution in dijets correlations in real and virtual photoproduction at HERA, Phys. Lett. B 500, 254 (2001) [arXiv:hep-ph/0011281].

[9] N. N. Nikolaev and B. G. Zakharov, Splitting the pomeron into two jets: A Novel process at HERA, Phys. Lett. B 332, 177 (1994) [arXiv:hep-ph/9403281].

[10] N. N. Nikolaev, W. Schäfer, B. G. Zakharov and V. R. Zoller, Nonlinear $k(T)$ factorization for forward dijets in DIS off nuclei in the saturation regime, J. Exp. Theor. Phys. 97, 441 (2003) [Zh. Eksp. Teor. Fiz. 124, 491 (2003)] [arXiv:hep-ph/0303024].

[11] N. N. Nikolaev, W. Schäfer and G. Schwiete, Coherent production of hard dijets on nuclei in QCD, Phys. Rev. D 63, 014020 (2000) [arXiv:hep-ph/0009038].

[12] N. N. Nikolaev, W. Schäfer, B. G. Zakharov and V. R. Zoller, Why breakup of photons and pions into forward dijets is so different: Predictions from nonlinear nuclear $k(T)$-factorization, Phys. At. Nucl. 68, 661 (2005) [Yad. Fiz. 68, 692 (2005)] [arXiv:hep-ph/0406085].

[13] N. N. Nikolaev and W. Schäfer, Breaking of k(T)-factorization for single jet production off nuclei, Phys. Rev. D 71, 014023 (2005) [arXiv:hep-ph/0411365]. 
[14] N. N. Nikolaev, W. Schäfer and B. G. Zakharov, Nonuniversality aspects of nonlinear $k(T)$-factorization for hard dijets, Phys. Rev. Lett. 95, 221803 (2005) [arXiv:hep-ph/0502018]; N. N. Nikolaev, W. Schäfer, B. G. Zakharov and V. R. Zoller, Nonlinear k(T)-factorization for quark-gluon dijet production off nuclei, Phys. Rev. D 72, 034033 (2005) [arXiv:hep-ph/0504057]; N. N. Nikolaev, W. Schäfer and B. G. Zakharov, Nonlinear $k(T)$-factorization for gluon-gluon dijets produced off nuclear targets, Phys. Rev. D 72, 114018 (2005) [arXiv:hep-ph/0508310].

[15] N. N. Nikolaev and W. Schäfer, Quenching of leading jets and particles: The $p(T)$ dependent Landau-Pomeranchuk-Migdal effect from nonlinear $k(T)$ factorization, Phys. Rev. D 74, 014023 (2006) [arXiv:hep-ph/0604117].

[16] N. N. Nikolaev and W. Schäfer, Unitarity cutting rules for the nucleus excitation and topological cross sections in hard production off nuclei from nonlinear $k(T)$-factorization, Phys. Rev. D 74, 074021 (2006) [arXiv:hep-ph/0607307].

[17] V. A. Abramovsky, V. N. Gribov and O. V. Kancheli, Character Of Inclusive Spectra And Fluctuations Produced In Inelastic Processes By Multi - Pomeron Exchange, Yad. Fiz. 18, 595 (1973) [Sov. J. Nucl. Phys. 18, 308 (1974)].

[18] J. P. Blaizot, F. Gelis and R. Venugopalan, High energy p A collisions in the color glass condensate approach. II: Quark production, Nucl. Phys. A 743, 57 (2004) [arXiv:hep-ph/0402257]; H. Fujii, F. Gelis and R. Venugopalan, Quantitative study of the violation of $k(T)$-factorization in hadroproduction of quarks at collider energies, Phys. Rev. Lett. 95, 162002 (2005) [arXiv:hep-ph/0504047]; J. Jalilian-Marian and Y. V. Kovchegov, Inclusive two-gluon and valence quark-gluon production in DIS and p A, Phys. Rev. D 70, 114017 (2004) [arXiv:hep-ph/0405266]; R. Baier, A. Kovner, M. Nardi and U. A. Wiedemann, Particle correlations in saturated QCD matter, Phys. Rev. D 72, 094013 (2005) [arXiv:hep-ph/0506126].

[19] R. J. Glauber, in Lectures in Theoretical Physics, edited by W. E. Brittin et al. (Interscience Publishers, Inc., New York, 1959), Vol. 1, p. 315.

[20] V. N. Gribov, Glauber Corrections And The Interaction Between High-Energy Hadrons And Nuclei, Sov. Phys. JETP 29, 483 (1969) [Zh. Eksp. Teor. Fiz. 56, 892 (1969)].

[21] N. N. Nikolaev and B. G. Zakharov, The Pomeron in diffractive deep inelastic scattering, J. Exp. Theor. Phys. 78, 598 (1994) [Zh. Eksp. Teor. Fiz. 105, 1117 (1994)][arXiv:hep-ph/9402324]; N. N. Nikolaev and B. G. Zakharov, The Triple pomeron regime and the structure function of the pomeron in the diffractive deep inelastic scattering at very small $x$, Z. Phys. C 64, 631 (1994) [arXiv:hep-ph/9306230]; N. N. Nikolaev, B. G. Zakharov and V. R. Zoller, The s channel approach to Lipatov's pomeron and hadronic cross-sections, JETP Lett. 59, 6 (1994) [arXiv:hep-ph/9312268].

[22] I. Balitsky, Operator expansion for high-energy scattering, Nucl. Phys. B 463, 99 (1996) [arXiv:hep-ph/9509348]; Y. V. Kovchegov, Small-x F2 structure function of a nucleus including multiple pomeron exchanges, Phys. Rev. D 60, 034008 (1999) [arXiv:hep-ph/9901281].

[23] A. Bialas, M. Bleszynski and W. Czyz, Multiplicity Distributions In Nucleus-Nucleus Collisions At High-Energies, Nucl. Phys. B 111, 461 (1976).

[24] A. Capella and A. Kaidalov, Hadron - Hadron And Hadron - Nucleus Scattering In Reggeon Calculus With Energy Momentum Conservation, Nucl. Phys. B 111, 477 (1976); L. Bertocchi and D. Treleani, Glauber Theory, Unitarity, And The Agk Cancellation, J. Phys. G 3, 147 (1977). 